\title{
Report
}

\section{LfDI Rheinland-Pfalz: 23. Tätigkeitsbericht 2010/2011}

Der Landesbeauftragte für den Datenschutz und die Informationsfreiheit, Edgar Wagner, hat am 14.02.2012 den Tätigkeitsbericht für die Jahre 2010/2011 dem Präsidenten des rheinland-pfälzischen Landtags, Joachim Mertes, überreicht.

Die Einführung zum Bericht beleuchtet die inhaltlichen Schwerpunkte:

In der digitalisierten Welt, in der wir überall Datenspuren hinterlassen, im Internet und außerhalb des Netzes, nimmt die Bedeutung des Datenschutzes zwangsläufig zu und damit auch die öffentliche Aufmerksamkeit an ihm.

Naturgemäß richtet sich die öffentliche Aufmerksamkeit im Lande auch nach den Ereignissen, die bundesweit Schlagzeilen machen. Im Berichtszeitraum waren dies nicht mehr die großen Datenskandale wie noch 2008 und 2009, als die Deutsche Bahn, die Deutsche Telekom, Lidl und andere deutsche Unternehmen für Aufregung sorgten. An ihre Stelle traten vielmehr Google, Facebook und Co., also die US-amerikanischen Internetgiganten, über deren Aktivitäten alle Medien intensiv berichteten, die elektronischen ebenso wie die Printmedien und bei diesen die seriösen Tageszeitungen ebensowie die Massenblätter. Facebook sorgte sogar für den Aufmacher bei der "Bild“-Zeitung und für einen Boykott-Aufruf im "Stern" („Raus aus Facebook"). Auch über Google Street View berichteten sämtliche Medien monatelang auf ihren politischen Seiten, in ihren politischen Magazinen und im Feuilleton. Diese Berichterstattung beförderte eine gesellschaftliche Diskussion über den Datenschutz, die glücklicherweise immer noch im Gang ist.

Der "Datenhunger" des Staates trat demgegenüber in den Hintergrund, wenn man von der Vorratsdatenspeicherung absieht, die Anfang 2010 in einer spektakulären Entscheidung des Bundesverfassungsgerichts gestoppt wurde und seither auch nicht wieder eingeführt worden ist. Datenschutzdefizite beim Einsatz des sog Staatstrojaners spielten zwar in der öffentlichen Diskussion ebenfalls noch eine gewisse Rolle. Im Übrigen herrschte aber eher Ruhe. Die neue Volkszählung wurde mit dem Segen des Bundesverfassungsgerichts und ohne nennenswerte Proteste in der Bevölkerung durchgeführt, der neue elektronische Personalausweis mit seinem RFID-Chip und besonderen Zusatzfunktionen mit kritischer Unterstützung der Datenschutzbeauftragten eingeführt und das ELENA-Verfahrensgesetz, das zu einer Vorratsdatenspeicherung zahlreicher Datensätze von 40 Millionen Beschäftigten geführt hätte, vom Bundestag still und leise aufgehoben.

Vor diesem Hintergrund spielte sich die datenschutzrelevante Entwicklung in Rheinland-Pfalz ab, jene im staatlichen Bereich und jene in der Wirtschaft. Weder in dem einen noch in dem anderen Bereich kam es zu großen Datenskandalen. Pannen und Datenschutzdefizite waren allerdings hier wie dort festzustellen. Immer mehr richtete sich die Aufmerksamkeit allerdings auf weitere Beteiligte: die Bürgerinnen und Bürger selbst, die zunehmend Daten von sich preisgeben, auch unbewusst Datenspuren hinterlassen und von der digitalen Entwicklung im Netz und außerhalb des Net- zes nicht selten überfordert werden, was nicht zuletzt auch darin zum Ausdruck kommt, dass die Bürgerinnen und Bürger sich immer häufiger hilfesuchend an meine Dienststelle wenden. Dabei ging und geht es auch um Fragen der Informationstechnik, zumal diese immer nachhaltiger in alle Lebensbereiche eindringt.

Der Bericht stellt diese Entwicklung dar und gibt Rechenschaft darüber, wie der LfD seine ihm übertragenen Aufgaben wahrgenommen hat. Er gliedert sich in zwei große Abschnitte. Im ersten Abschnitt finden sich grundsätzliche Anmerkungen zum Stellenwert des Datenschutzes, zur Entwicklung des Datenschutzrechts und zu den thematischen Schwerpunkten des Datenschutzes sowie zum Datenschutz als Bildungs- und Erziehungsaufgabe. Im zweiten Abschnitt werden ausgewählte Prüfungsergebnisse zusammengefasst. Der Bericht endet mit einigen Bemerkungen zur Dienststelle des LfD und ihrer Entwicklung im Berichtszeitraum.

Der Tätigkeitsbericht ist auf der Website $h t t p: / / w w w . d a t e n s c h u t z$. rlp.de/de/ds.php?submenu=bericht verfügbar.

\section{LfDI Brandenburg: 16. Tätigkeitsbericht 2010/2011}

Die Landesbeauftragte für den Datenschutz und für das Recht auf Akteneinsicht, Dagmar Hartge, veröffentlichte am 06.03.2012 den 16. Tätigkeitsbericht für die Jahre 2010 und 2011. Es ist der erste Bericht seit der Zusammenführung der Datenschutzaufsicht über den öffentlichen und privaten Bereich.

Hartge: „Datenschutzverstöße von Unternehmen - das hat mir die neue Zuständigkeit für die Datenschutzaufsicht über den privaten Bereich gezeigt - sind für die Bürgerinnen und Bürger mindestens ebenso schwerwiegend, wie Verstöße öffentlicher Stellen."

Hartges Resümee spiegelt sich in den Schwerpunkten des aktuellen Tätigkeitsberichts wider. So erreichten ihre Behörde beispielsweise zahlreiche Beschwerden über die Videoüberwachung des öffentlichen Raums oder in Unternehmen.

Die Vorbereitung und Durchführung des Zensus 2011 wurde von der Landesbeauftragten im Berichtszeitraum ausführlich begleitet. Bei ihren Kontrollen konnte sie sich von der datenschutzgerechten Abschottung der Erhebungsstellen von der regulären Verwaltung ebenso überzeugen wie vom ordnungsgemäßen Umgang mit den erhobenen Unterlagen. Die erforderlichen technischen Sicherheitsmaßnahmen wurden sowohl in den Erhebungsstellen vor Ort als auch im Amt für Statistik Berlin-Brandenburg ergriffen.

Dass der datenschutzgerechte Umgang mit sensitiven Patientendaten im Gesundheitswesen eine wichtige Voraussetzung für das Vertrauen der Patientinnen und Patienten darstellt, gilt sowohl für Amtsärzte als auch für private Praxen.

Dagmar Hartge:

„Besonders bei privaten Kliniken oder Arztpraxen besteht in Sachen Datenschutz und Datensicherheit noch erheblicher Nachholbedarf. Die Begleitung neuer Projekte zur Telemedizin sowie Kon- 